thấy lo lắng khi xem truyền thông $(\mathrm{OR}=4,4$; 95\%CI: $1,9-10,2$ )

\section{TÀl LIẸU THAM KHẢO}

1. Cục Y tế Dự phòng, Bộ Y Tế (2020). COVID-19 DASHBOARD. $h$ https://ncov.vncdc.gov.vn/>, accessed: 30/05/2020.

2. Chew N.W.S., Lee G.K.H., Tan B.Y.Q. và công sứ. (2020). A multinational, multicentre study on the psychological outcomes and associated physical symptoms amongst healthcare workers during COVID-19 outbreak. Brain, Behavior, and Immunity.

3. Pappa S., Ntella V., Giannakas T. và cộng sự. (2020). Prevalence of depression, anxiety, and insomnia among healthcare workers during the COVID-19 pandemic: A systematic review and meta-analysis. Brain Behav Immun.

4. Lai J., Ma S., Wang Y. và cộng sự. (2020). Factors Associated With Mental Health Outcomes
Among Health Care Workers Exposed to Coronavirus Disease 2019. JAMA Netw Open, 3(3).

5. National Institutes of Health (2019). PostTraumatic Stress Disorder. <https://www.nimh.nih.gov/health/topics/posttraumatic-stress-disorder-ptsd/index.shtml>, accessed: 15/05/2020.

6. Tan B.Y.Q., Chew N.W.S., Lee G.K.H. và cộng sư. (2020). Psychological Impact of the COVID19 Pandemic on Health Care Workers in Singapore. Ann Intern Med.

7. WHO (2017), Depression and Other Common Mental Disorders, World Health Organization.

8. Lai J., Ma S., Wang Y. và công sứ. (2020). Factors Associated With Mental Health Outcomes Among Health Care Workers Exposed to Coronavirus Disease 2019. JAMA Netw Open, 3(3).

9. Zhang W., Wang $K .$, Yin $L$. và cộng sự. (2020). Mental Health and Psychosocial Problems of Medical Health Workers during the COVID-19 Epidemic in China. Psychother Psychosom, 1-9.

\title{
MộT SỐ ĐĂC ĐIỂM CẤU TRÚC SO MĂT Ở TRẺ EM NGƯờI KINH TỪ 7-9 TUỔI TRÊN PHIM SỌ NGHIÊ̂NG THEO PHÂN TÍCH RICKETTS
}

\section{Trương Đình Khởi ${ }^{1}$, Lương Ngọc Khuê ${ }^{2}$, Đào Thị Dung ${ }^{3}$, Hà Ngọc Chiều ${ }^{1}$, Đinh Diệu Hồng ${ }^{3}$ \\ CRANIOFACIAL CHARACTERISTICS IN KINH ETHNIC CHILDREN FROM 7 TO 9 YEARS OF AGE ON LATERAL CEPHALOGRAMS USING RICKETTS ANALYSIS \\ Objectives: To determine craniofacial}

\section{TÓM TẮT}

Muc tiêu: Xác định một số đăc điểm so mă̆t và phân tích sự tăng trưởng đâu mặ̆t từ 7-9 tuổi trên phim sọ nghiêng sử dụng phân tích của Ricketts. Đối tượng và phương pháp nghiên cứu: Nghiên cứu dọc trên 206 trẻ $7-9$ tuổi người Kinh tại trường Tiểu học Liên Ninh, Thanh trì, Hà Nội bằng phương pháp đo trên phim so nghiêng kỹ thuất số. Kết quả: Chiều cao mặt toàn bộ (Ba-N/Xi-Pm), chiều cao tầng mặt dưới (Ans-Xi-Pm), góc măt phẳng hàm dưới (Go$\mathrm{Me} / \mathrm{Fh}$ ) sự khác biệt không có ý nghĩa thống kê giữa nam và nữ ở trẻ 7 tuổi, nhưng ở loai III lớn hơn loai I và II Angle. Chiều cao mặt toàn bộ và chiều cao mặt dưới không đổi ở loại I Angle, tăng trưởng đều đặn ở loại II và III Angle từ 7-9 tuổi. Góc mặt phẳng hàm dưới giảm theo tuổi ở loại I Angle, tăng dần theo tuổi ở loại II và III Angle. Kết luận: Các chỉ số đặc điểm so mắt ở trẻ 7 tuổi người Kinh giống nhau giửa nam và nữ, xu hướng nữ tăng trưởng sớm hơn nam.

Tư khóa: Chiều cao mặt toàn bộ, chiều cao mặt dưới, góc mặt phẳng hàm dưới

\section{SUMMARY}

${ }^{1}$ Viện Đào tạo Răng Hàm Mặt- Đại học Y Hà Nội ${ }^{2}$ Cục Khám chữa bệnh và Cục Y tế dự phòng-Bộ y Tế,

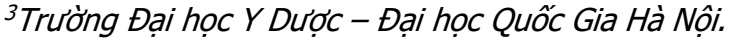
Chiu trách nhiếm chính: Trương Đình Khởi

Email: bskhoirhm@gmail.com

Ngày nhận bài: 11.6.2021

Ngày phản biện khoa họ: 2.8.2021

Ngày duyệt bài: 12.8.2021 characteristics and evaluate the growth of Kinh ethnic children from 7 to 9 years old on lateral cephalograms. Subjects and methods: A longitudinal study of 206 children (104 males, 102 females) from 7 to 9 years old in Lien Ninh primary school, Thanhtri, Hanoi by measuring on lateral cephalograms according to Ricketts analysis. Results: Average of total facial height (Ba-N/Xi-Pm), lower facial height (Ans-Xi-Pm) and mandibular plan angle (Go-Me/Fh) were no difference between male and female in 7 years old children, but in class III was larger in class I and II Angle. Conclusion: Most of craniofacial dimensions were no difference between male and female, craniofacial growth in female was significantly sooner than in male.

Keywords: total facial height, lower facial height, mandibular plan angle

\section{I. ĐĂT VẤN ĐỀ}

Chỉnh hình răng mặt trở thành nhu câu của xã hội, trong đó, từ 7-9 tuổi là thời điểm quan trọng trong điêu trị dự phòng và can thiệp sớm, nhờ vào đánh giá đặc điểm nhân trắc đâuu mặt mà các bác sỹ lâm sàng có thể hiểu rõ hơn tình trạng bệnh lý, tiên lượng được xu hướng tăng trưởng để quyết định kế hoạch điêu trị và có thể 
hình dung được khuôn mặt trong tương lai về chiều cao, chiều rộng và chiều ngang.

Trên thế giới đã có nhiều nghiên cứu cấu trúc sọ mặt trên phim sọ nghiêng ở nhiều lứa tuổi khác nhau. Nghiên cứu của Ricketts R.M (1996) [1], đưa ra phương pháp phân tích phim sọ mặt có nhiêu ý nghĩa quan trọng khi nghiên cứu sự tăng trưởng của kết cấu sọ mặt, đưa ra được các chỉ số vùng đầu mặt ở trẻ 9 tuổi và những dự đoán khoảng tăng trưởng ở người Caucasian. Nghiên cứu của Plotou.C (1983) [2] thực hiện đo đạc theo phân tích Ricketts trên 30 trẻ em 12 tuổi tại Oslo, Na Uy. Công trình nghiên cứu của Tae Soo Park và cộng sự (1983) [3] thực hiện nghiên cứu dọc trền 90 đối tượng nghiên cứu (40 nam, 50 nữ) trẻn em Hàn Quốc từ 6 đến 9 tuổi theo phân tích Ricketts. Công trình nghiên cứu của Hideyuki Kato, Satoshi Fujii và cộng sự (1988) [4] nghiên cứu thực hiện trên 221 đổi tượng trẻ em người Nhật Bản từ 3 đến 10 tuổi.

Tại Việt Nam, đã có một số nghiên cứu như công trình nghiên cứu của Lê Võ Yến Nhi, Hoàng Tử Hùng (2011) [5], nghiên cứu dọc trên 39 đối tượng (19 nam, 20 nữ) từ 10 đến 14 tuổi. Nghiên cứu của Lê Nguyên Lâm (2014) [6], nghiên cứu dọc trên phim sọ nghiêng theo phân tích Ricketts 105 trẻ (50 nam và 55 nữ) trẻ từ 12-15 tuổi. Nghiên cứu của Phạm Cao Phong (2016) [7] nghiên cứu một số chỉ số sọ mặt ở 122 học sinh (62 nam, 60 nữ) người Việt từ 11 đến 13 tuổi Như vậy các nghiên cứu theo phân tích Ricketts còn ít, trong đó chưa có nghiên cứu từ 7-9 tuổi. Chính vì vậy, chúng tôi tiến hành nghiên cứu đề tài "Môt số đặc điểm câuu trúc sọ mặt ở trẻ em người Kinh từ 7-9 tuổi trên phim sọ nghiêng theo phân tích Ricketts "với mục tiêu: Xác định một số đặc điểm so mặt và sự tăng trưởng ở trẻ người Kinh 7 đến 9 tuôii tại Hà Nội theo phân tích Ricketts.

\section{II. ĐỐI TƯớNG VÀ PHƯƠ'NG PHÁP NGHIÊN CỨU}

2.1. Đối tượng nghiên cứu. Là 206 trẻ em người Kinh 7 tuổi bao gồm 104 nam và 102 nữ.

- Tiêu chuấn lựa chọn:

- Đối tượng là người dân tộc Kinh, có bố mẹ, ông bà nội ngoại là người Kinh, không điều trị chỉnh hình răng mặt trước và trong thời gian nghiên cứu, không có dị tật bẩm sinh, không có biến dạng xương hàm, không mắc bệnh ảnh hưởng đến tăng trưởng của cơ thể và vùng đầu - mặt, không có viêm nhiễm hoặc chấn thương nghiêm trọng vùng hàm mặt, trẻ và người thân của trẻ (cha mẹ hoặc người giám hộ) đồng ý tham gia nghiên cứu.
- Đã mọc đầy đủ đủ bốn răng hàm lớn thứ nhất và chạm khớp hai hàm, khớp cắn theo phân loại Angle hai bên trái và phải giống nhau thể hiện cùng phân loại khớp cắn loại I, II và III Angle.

- Tiêu chuân loại trư: Đối tượng không đủ các tiêu chuẩn lựa chọn.

- Thời gian và địa điểm nghiên cứu: Từ tháng 10/2017 đến tháng 10/2020 tại trường Tiểu học Liên Ninh, Thanh Trì, Hà Nội.

\subsection{Phương pháp nghiên cứu}

- Thiết kế nghiên cứu: Nghiên cứu dọc

- Vật liệu và phương tiện nghiên cứu: Máy chụp phim X Quang kỹ thuật số Orthophos XG5, hãng sản xuất: Sirona, 60-84KV, 3-15mA, thời gian chiếu xạ $0.16 \mathrm{~s}-2.5 \mathrm{~s}$, liều chiếu xạ cho mỗi lần chụp < $0,003 \mathrm{mSv}$, đời máy chụp đa năng Orthophos XG5 là một trong những máy hiện đại nhất.

- Các điểm mốc giải phẫu [1],[5],[6]: Điểm cao nhất của lỗ ống tai ngoài (Porion- Po), điểm trước nhất của khớp trán - mũi (Nasion-N), điểm dưới nhất của bờ trước lố chẩm (Basion-Ba), điểm thấp nhất của bờ dưới ổ mắt (Orbitale-Or), điểm trung tâm của góc hàm xương hàm dưới (Xi point-Xi), điểm trước nhất của gia mũi trước (Anterior nasale spine-Ans), điểm sau và thấp nhất của góc hàm (Gonion-Go), điểm cằm trên, tiếp giáp đường viền cằm phía trước và vùng bao túi cằm (Suprapogonion-Pm), điểm trước nhất của vùng cằm (Pogonion-Pog), điểm thấp nhất của vùng cằm (Mention-Me), mặt phẳng Frankfort (Fh): Đi qua hai điểm Po và Or trên phim so nghiêng.

- Các kích thước đo đạc [1],[5],[6]: Chiều cao mặt toàn bộ $(\mathrm{Ba}-\mathrm{N} / \mathrm{Xi}-\mathrm{Pm})$, chiêu cao mặt dưới (Ans-Xi-Pm), góc mặt phẳng hàm dưới (Go$\mathrm{Me} / \mathrm{Fh})$.

- Xử lý số liệu: Số liệu sau khi thu thập được nhập liệu và xử lý bằng phần mềm SPSS 23.0. Khi cần so sánh giá trị trung bình giữa hai giới hoặc hai nhóm sử dụng t-test hoặc MannWhitney test. Khi so sánh mức độ tăng trưởng và tỷ lệ tăng trưởng của hai nhóm tuổi 7-8 và $8-9$ của từng loại khớp cắn thì sử dụng so sánh bắt cặp (paired - samples t test) hoặc Wilcoxon test; khi so sánh giá trị trung bình cùng giới giữa các loại khớp cắn thì sử dụng one way Anova test kểt hợp Bonferoni test hoặc Kruskal Wallis test kết hợp Mann- Whitney test.

- Đạo đức trong nghiên cứu: Tiến hành nghiên cứu trên các đối tượng tự nguyện tham gia nghiên cứu, được Hội đồng đạo đức $Y$ sinh học của trường Đại học Y Hà Nội cấp giấy chấp thuận số 47/HĐĐĐĐHYHN ngày 12/01/2017. 


\section{KẾT QUẢ NGHIÊN CứU}

Nghiên cứu 206 đối tượng trẻ em 7 tuổi dân tộc Kinh bao gồm 104 nam và 102 nữ bằng phương pháp đo trên phim sọ nghiêng cho kết quả như sau:

Bảng 3.1: So sánh giữa ba loại khớp căn giá trị trung bình chiều cao măt toàn bộ, chiều cao tầng mặt dưới và góc mặt phẳng hàm dưới ở trẻ em người Kinh 7 tuối

\begin{tabular}{|c|c|c|c|c|c|c|}
\hline \multirow{2}{*}{\multicolumn{2}{|c|}{$\begin{array}{c}\text { Kích thước } \\
\text { đâu mặt }\end{array}$}} & \multicolumn{4}{|c|}{ Phân loại khớp căn theo Angle } & \multirow{3}{*}{$\begin{array}{c}\mathbf{P} \\
\begin{array}{c}\mathrm{P}_{\mathrm{I}-\mathrm{II}} ; \mathrm{P}_{\mathrm{I}-\mathrm{III}} ; \mathrm{P}_{\mathrm{II}-\mathrm{III}} \\
<0,017\end{array}\end{array}$} \\
\hline & & Loại I & Loại II & Loại III & Chung & \\
\hline \multirow{4}{*}{$\begin{array}{c}\text { Ba-N/ } \\
\text { Xi-pm } \\
\left({ }^{\circ}\right)\end{array}$} & Nam & $59,98 \pm 3,02$ & $55,89 \pm 2,85$ & $66,53 \pm 3,04$ & $60,68 \pm 5,25$ & \\
\hline & Nũ̃ & $59,68 \pm 2,88$ & $55,95 \pm 2,82$ & $65,86 \pm 3,03$ & $60,41 \pm 4,94$ & $\begin{array}{c}\mathrm{P}_{\text {I-III }} \mathrm{P}_{\text {I-III }} ; \mathrm{P}_{\text {II-III }}<0,017 \\
\end{array}$ \\
\hline & $\mathrm{P}_{1}$ & 0,6651 & 0,9382 & 0,3767 & 0,7025 & \\
\hline & Chung & $59,83 \pm 2,93$ & $55,92 \pm 2,81$ & $66,20 \pm 3,03$ & $60,55 \pm 5,09$ & $\begin{array}{c}\mathrm{P}_{\mathrm{I}-\mathrm{III}} ; \mathrm{P}_{\mathrm{I}-\mathrm{III}} ; \mathrm{P}_{\mathrm{II}-\mathrm{III}} \\
<0,017\end{array}$ \\
\hline \multirow{4}{*}{$\begin{array}{c}\text { Ans-Xi- } \\
\text { Pm } \\
\left({ }^{\circ}\right)\end{array}$} & Nam & $46,36 \pm 2,98$ & $41,86 \pm 2,89$ & $50,41 \pm 2,99$ & $46,13 \pm 4,54$ & $\begin{array}{c}\text { PI-II; } P_{\text {I-III }} ; P_{\text {II-III }} \\
<0,017\end{array}$ \\
\hline & Nũ̃ & $46,22 \pm 2,92$ & $41,17 \pm 2,85$ & $51,46 \pm 3,06$ & $46,23 \pm 5,05$ & $\begin{array}{c}\text { PI-II; PI-III; PII-III } \\
<0,017\end{array}$ \\
\hline & $\mathrm{P}_{1}$ & 0,8442 & 0,3270 & 0,1679 & 0,8809 & \\
\hline & Chung & $46,29 \pm 2,93$ & $41,52 \pm 2,87$ & $50,93 \pm 3,04$ & $46,18 \pm 4,79$ & $\begin{array}{c}\text { PI-II; } \text { PI-IIII; }^{<} \text {PII-III } \\
<0,017\end{array}$ \\
\hline \multirow{4}{*}{$\begin{array}{c}\text { Go- } \\
\mathrm{Me} / \mathrm{Fh} \\
\left(^{\circ}\right)\end{array}$} & Nam & $25,16 \pm 2,97$ & $22,88 \pm 3,19$ & $28,79 \pm 2,94$ & $25,54 \pm 3,85$ & $\begin{array}{c}\mathrm{P}_{\text {I-II }} \mathrm{P}_{\text {I-III }} ; \mathrm{P}_{\text {II-III }} \\
<0,017\end{array}$ \\
\hline & Nũ̃ & $25,29 \pm 2,98$ & $22,99 \pm 3,25$ & $29,26 \pm 3,24$ & $25,79 \pm 3,96$ & $\begin{array}{c}\mathrm{P}_{\text {I-II; }} \mathrm{P}_{\text {I-III }} ; \mathrm{P}_{\text {IIIIII }} \\
<0,017\end{array}$ \\
\hline & $\mathrm{P}_{1}$ & 0,8505 & 0,8851 & 0,5434 & 0,7917 & \\
\hline & Chung & $25,22 \pm 2,95$ & $22,93 \pm 3,19$ & $29,02 \pm 3,07$ & $25,66 \pm 3,90$ & 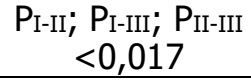 \\
\hline
\end{tabular}

(p1: Sample T-test; p: One way ANOVA kết hợp Bonferoni test)

Nhận xét: Theo kết quả nghiên cứu, chiều cao mặt toàn bộ (Ba-N/Xi-Pm), chiều cao tầng mặt dưới (Ans-Xi-Pm), góc mặt phẳng hàm dưới (Go-Me/Fh) sự khác biệt không có ý nghĩa thống kê giữa hai giới ở mỗi loại khớp cắn với $p>0,05$; khi so sánh cùng giới, các kích thước loại III Angle lớn hơn loại I và loại II Angle với $p<0,017$.

Bảng 3.2: Mức tăng trưởng và tỷ lệ gia tăng chiều cao mặt toàn bộ bằng phương pháp đo trên phim sọ nghiêng từ 7-9 tuối

\begin{tabular}{|c|c|c|c|c|c|c|}
\hline \multirow{2}{*}{\multicolumn{3}{|c|}{ 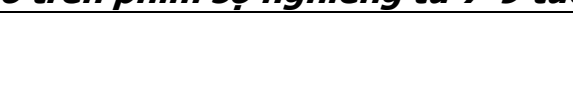 }} & \multicolumn{3}{|c|}{ Đố tuối } & \multirow[b]{2}{*}{ p } \\
\hline & & & $7-8$ & $8-9$ & $7-9$ & \\
\hline \multirow{8}{*}{ Loại II } & \multirow{4}{*}{$\begin{array}{l}\text { Mức gia } \\
\text { tăng (mm) }\end{array}$} & Nam & $0,50 \pm 0,32$ & $0,57 \pm 0,33$ & $1,07 \pm 0,42$ & $0,9087^{* *}$ \\
\hline & & Nữ & $0,51 \pm 0,31$ & $0,58 \pm 0,35$ & $1,09 \pm 0,43$ & $0,1951^{* *}$ \\
\hline & & $\mathrm{P}_{1}$ & $0,3668^{* *}$ & $0,3635^{* *}$ & $0,5849 * *$ & \\
\hline & & Chung & $0,51 \pm 0,31$ & $0,58 \pm 0,34$ & $1,08 \pm 0,42$ & $0,2918^{* *}$ \\
\hline & \multirow{4}{*}{$\begin{array}{l}\text { Tỷ lê gia } \\
\text { tăng (\%) }\end{array}$} & Nam & $0,89 \pm 0,53$ & $1,03 \pm 0,46$ & $1,94 \pm 0,73$ & $0,6702^{* *}$ \\
\hline & & Nữ & $0,91 \pm 0,60$ & $1,06 \pm 0,41$ & $1,98 \pm 0,79$ & $0,3127^{* *}$ \\
\hline & & $P_{1}$ & $0,3447 * *$ & $0,6410 * *$ & $0,6631^{* *}$ & \\
\hline & & Chung & $0,90 \pm 0,56$ & $1,05 \pm 0,43$ & $1,96 \pm 0,75$ & $0,5534 * *$ \\
\hline \multirow{8}{*}{ Loại III } & \multirow{4}{*}{$\begin{array}{l}\text { Mức gia } \\
\text { tăng (mm) }\end{array}$} & Nam & $0,94 \pm 0,28$ & $0,98 \pm 0,26$ & $1,92 \pm 0,29$ & $0,5554 * *$ \\
\hline & & Nữ & $0,96 \pm 0,29$ & $0,99 \pm 0,28$ & $1,95 \pm 0,27$ & $0,5621^{* *}$ \\
\hline & & $P_{1}$ & $0,8645^{* *}$ & $0,8029 * *$ & $0,8183^{* *}$ & \\
\hline & & Chung & $0,95 \pm 0,28$ & $0,98 \pm 0,27$ & $1,93 \pm 0,28$ & $0,3937 * *$ \\
\hline & \multirow{4}{*}{$\begin{array}{l}\text { Tỷ lẹ gia } \\
\text { tăng (\%) }\end{array}$} & Nam & $1,41 \pm 0,43$ & $1,45 \pm 0,40$ & $2,89 \pm 0,47$ & $0,6616^{* *}$ \\
\hline & & Nũ & $1,46 \pm 0,44$ & $1,48 \pm 0,44$ & $2,97 \pm 0,49$ & $0,7506 * *$ \\
\hline & & $\mathrm{P}_{1}$ & $0,8133^{* *}$ & $0,2999 * *$ & $0,4235^{* *}$ & \\
\hline & & Chung & $1,44 \pm 0,43$ & $1,47 \pm 0,42$ & $2,93 \pm 0,48$ & $0,6403 * *$ \\
\hline
\end{tabular}

p1, p (*: sample t-test, **: Mann - Whitney test) 
Nhận xét: Mức gia tăng và tỷ lệ gia tăng chiều cao mặt toàn bộ sự khác biệt không có ý nghĩa thống kê giữa hai giới ở từng khoảng tuổi tăng trưởng. Mức gia tăng và tỷ lệ gia tăng từ 8-9 tuổi lớn hơn từ 7-8 tuổi, tuy nhiên, sự khác biệt không có ý nghĩa thống kê (p>0,05).

Bảng 3.3: Mức tăng trưởng và tỷ lệ gia tăng chiều cao mặt dưới bằng phương pháp đo trên phim sọ nghiêng từ 7-9 tuổi

\begin{tabular}{|c|c|c|c|c|c|c|}
\hline \multirow{2}{*}{\multicolumn{3}{|c|}{ 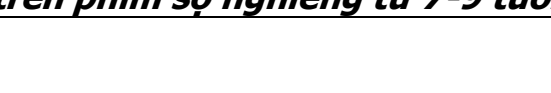 }} & \multicolumn{3}{|c|}{ Độ Tuối } & \multirow[b]{2}{*}{$\mathbf{p}$} \\
\hline & & & $7-8$ & $8-9$ & 7-9 & \\
\hline \multirow{8}{*}{ Loại II } & \multirow{4}{*}{$\begin{array}{l}\text { Mức gia } \\
\text { tăng (mm) }\end{array}$} & Nam & $0,40 \pm 0,25$ & $0,50 \pm 0,28$ & $0,90 \pm 0,36$ & $0,0457 * *$ \\
\hline & & Nữ & $0,50 \pm 0,26$ & $0,62 \pm 0,24$ & $1,12 \pm 0,29$ & $0,4915^{* *}$ \\
\hline & & $\mathrm{P}_{1}$ & $0,5849 * *$ & $0,4503^{* *}$ & $0,4652 * *$ & \\
\hline & & Chung & $0,46 \pm 0,26$ & $0,57 \pm 0,26$ & $1,03 \pm 0,33$ & $0,0677 * *$ \\
\hline & \multirow{4}{*}{$\begin{array}{l}\text { Tỷ lệ gia } \\
\text { tăng (\%) }\end{array}$} & $\mathrm{Nam}$ & $0,96 \pm 0,65$ & $1,18 \pm 0,67$ & $2,15 \pm 0,75$ & $0,0797 * *$ \\
\hline & & Nữ & $1,21 \pm 0,70$ & $1,49 \pm 0,72$ & $2,73 \pm 0,81$ & $0.6487 * *$ \\
\hline & & $\mathrm{P}_{1}$ & $0,5194 * *$ & $0,5935^{* *}$ & $0,3736^{* *}$ & \\
\hline & & Chung & $1,03 \pm 0,67$ & $1,36 \pm 0,65$ & $2,45 \pm 0,77$ & $0.1528^{* *}$ \\
\hline \multirow{8}{*}{ Loại III } & \multirow{4}{*}{$\begin{array}{l}\text { Mức gia } \\
\text { tăng (mm) }\end{array}$} & Nam & $0,85 \pm 0,31$ & $0,93 \pm 0,32$ & $1,78 \pm 0,40$ & $0,0948^{* *}$ \\
\hline & & Nữ & $0,85 \pm 0,27$ & $0,94 \pm 0,26$ & $1,79 \pm 0,33$ & $0,0683 * *$ \\
\hline & & $P_{1}$ & $0,9477^{* *}$ & $0,9059^{* *}$ & $0,8852^{* *}$ & \\
\hline & & Chung & $0,84 \pm 0,29$ & $0,94 \pm 0,29$ & $1,78 \pm 0,36$ & $0,0128 * *$ \\
\hline & \multirow{4}{*}{$\begin{array}{l}\text { Tỷ lệ gia } \\
\text { tăng (\%) }\end{array}$} & Nam & $1,69 \pm 0,66$ & $1,81 \pm 0,63$ & $3,53 \pm 0,85$ & $0,1773^{* *}$ \\
\hline & & Nữ & $1,65 \pm 0,50$ & $1,80 \pm 0,54$ & $3,48 \pm 0,66$ & $0,0757^{* *}$ \\
\hline & & $\mathrm{P}_{1}$ & $0,8440 * *$ & $0,8031^{* *}$ & $0,6746 * *$ & \\
\hline & & Chung & $1,67 \pm 0,59$ & $1,80 \pm 0,58$ & $3,50 \pm 0,76$ & $0,0274 * *$ \\
\hline
\end{tabular}

$\mathrm{p} 1, \mathrm{p}(*$ : sample t-test, $* *$ : Mann - Whitney test)

Nhận xét: Mức gia tăng và tỷ lệ gia tăng chiêu cao mặt dưới sự khác biệt không có ý nghĩa thống kê giữa hai giới khoảng từ 7-8 tuổi và 8-9 tuổi ở khớp cắn loại II và III Angle. Sự khác biệt mức gia tăng và tỷ lệ gia tăng chiều cao mặt dưới từ 7-8 tuổi và 8-9 tuổi cùng giới và cùng loại khớp cắn không có ý nghĩa thống kê.

Bảng 3.4: Mức tăng trưởng và tỷ lệ gia tăng góc mặt phẳng hàm dưới bằng phương pháp đo trên phim sọ nghiêng từ 7-9 tuối

\begin{tabular}{|c|c|c|c|c|c|c|}
\hline & \multicolumn{3}{|c|}{ Độ Tuối } & \multirow[b]{2}{*}{$\mathbf{p}$} \\
\hline & & & $7-8$ & 8-9 & $7-9$ & \\
\hline \multirow{8}{*}{ Loại I } & \multirow{4}{*}{$\begin{array}{c}\text { Mức gia } \\
\text { tăng (mm) }\end{array}$} & Nam & $-0,48 \pm 0,40$ & $-0,51 \pm 0,32$ & $-0,99 \pm 0,38$ & $0,8752^{* *}$ \\
\hline & & Nữ & $-0,49 \pm 0,46$ & $-0,52 \pm 0,37$ & $-1,01 \pm 0,39$ & $0,5512^{* *}$ \\
\hline & & $P_{1}$ & $0,8355^{* *}$ & $0,7826^{* *}$ & $0,8048 * *$ & \\
\hline & & Chung & $-0,49 \pm 0,44$ & $-0,52 \pm 0,35$ & $-1,01 \pm 0,39$ & $0,7708^{* *}$ \\
\hline & \multirow{4}{*}{$\begin{array}{l}\text { Tỷ lệ gia } \\
\text { tăng }(\%)\end{array}$} & Nam & $-1,91 \pm 1,68$ & $-2,07 \pm 1,47$ & $-3,94 \pm 1,76$ & $0,7534^{* *}$ \\
\hline & & Nữ & $-1,94 \pm 1,74$ & $-2,09 \pm 1,48$ & $-3,99 \pm 1,84$ & $0,5412^{* *}$ \\
\hline & & $P_{1}$ & $0,8651^{* *}$ & $0,7954 * *$ & $0,7955^{* *}$ & \\
\hline & & Chung & $-1,93 \pm 1,71$ & $-2,08 \pm 1,47$ & $-3,97 \pm 1,81$ & 0,8929** \\
\hline \multirow{8}{*}{ Loại II } & \multirow{4}{*}{$\begin{array}{c}\text { Mức gia } \\
\text { tăng (mm) }\end{array}$} & Nam & $0,40 \pm 0,38$ & $0,42 \pm 0,35$ & $0,82 \pm 0,43$ & $0,9804 * *$ \\
\hline & & Nũ̃ & $0,42 \pm 0,39$ & $0,49 \pm 0,38$ & $0,91 \pm 0,48$ & $0,6359 * *$ \\
\hline & & $P_{1}$ & $0,8251^{* *}$ & $0,4395^{* *}$ & $0,4728^{* *}$ & \\
\hline & & Chung & $0,41 \pm 0,38$ & $0,46 \pm 0,37$ & $0,87 \pm 0,46$ & $0,7833 * *$ \\
\hline & \multirow{4}{*}{$\begin{array}{l}\text { Tỷ lệ gia } \\
\text { tăng }(\%)\end{array}$} & Nam & $1,75 \pm 1,28$ & $1,80 \pm 1,31$ & $3,58 \pm 1,79$ & $0,8828^{* *}$ \\
\hline & & Nũ̃ & $1,83 \pm 1,36$ & $2,09 \pm 1,42$ & $3,96 \pm 1,85$ & $0,7141^{* *}$ \\
\hline & & $\mathrm{P}_{1}$ & $0,8348 * *$ & $0,4006^{* *}$ & $0,411^{* *}$ & \\
\hline & & Chung & $1,80 \pm 1,32$ & $1,97 \pm 1,38$ & $3,79 \pm 1,83$ & $0,9318^{* *}$ \\
\hline \multirow{6}{*}{ Loại III } & \multirow{4}{*}{$\begin{array}{c}\text { Mức gia } \\
\text { tăng (mm) }\end{array}$} & Nam & $0,47 \pm 0,29$ & $0,52 \pm 0,31$ & $0,99 \pm 0,38$ & $0,9501 * *$ \\
\hline & & Nữ & $0,49 \pm 0,32$ & $0,54 \pm 0,34$ & $1,03 \pm 0,46$ & $0,7224 * *$ \\
\hline & & $P_{1}$ & $0,5288^{* *}$ & $0,3793^{* *}$ & $0,4084 * *$ & \\
\hline & & Chung & $0,48 \pm 0,31$ & $0,53 \pm 0,33$ & $1,01 \pm 0,43$ & $0,8677 * *$ \\
\hline & \multirow{2}{*}{$\begin{array}{l}\text { Tỷ lệ gia } \\
\text { tăng (\%) }\end{array}$} & Nam & $1,63 \pm 1,27$ & $1,78 \pm 1,32$ & $3,44 \pm 1,66$ & $0,9929 * *$ \\
\hline & & Nữ & $1,67 \pm 1,33$ & $1,82 \pm 1,36$ & $3,52 \pm 1,73$ & $0,6536 * *$ \\
\hline
\end{tabular}




\begin{tabular}{|c|c|c|c|c|c|c|}
\hline & $\mathrm{P}_{1}$ & $0,4868^{* *}$ & $0,3722^{* *}$ & $0,5034 * *$ & \\
\cline { 3 - 7 } & & Chung & $1,65 \pm 1,31$ & $1,81 \pm 1,34$ & $3,48 \pm 1,69$ & $0,7762 * *$ \\
\hline
\end{tabular}

p1, p (*: sample t-test, **: Mann - Whitney test)

Nhân xét: Theo kết quả nghiên cứu, mức gia tăng và tỷ lệ gia tăng góc mặt phẳng hàm dưới sự khác biệt không có ý nghĩa thống kê giữa hai giới ở từng khoảng tuổi tăng trưởng (từ 7-8 tuổi, $8-9$ tuổi và 7-9 tuổi) với $p>0,05$. Khi so sánh mức gia tăng và tỷ lệ gia tăng góc mặt phẳng hàm dưới từ 7-8 tuổi và 8-9 tuổi trong cùng giới tính và cùng loại khớp cắn thì từ 8-9 tuổi lớn hơn từ 7-8 tuổi, tuy nhiên, sự khác biệt không có ý nghĩa thống kê $(p>0,05)$.

\section{BÀN LUÂN}

Nghiên cứu 206 đối tượng trẻ em 7 tuổi dân tộc Kinh bao gồm 104 nam và 102 nữ bằng phương pháp đo trên phim sọ nghiêng theo phân tích Ricketts, chúng tôi có một số bàn luận như sau:
Chiêu cao mặt toàn bộ. Theo kết quả bảng 3.1, chiều cao mặt toàn bộ sự khác biệt không có ý nghĩa thống kê giữa hai giới nhưng ở loại III Angle lớn hơn loại I Angle và loại I Angle lớn hơn loại II Angle, loại III Angle có xu hướng góc mơ hơn so với loại I và II Angle. Khi so sánh với nghiên cứu của Ricketts [1] và Eun Ju Bae, Hye Jin Kwon, Oh Won Kwon và công sứ (2014) [8], cho thấy trẻ người Kinh 7 tuổi có chiều mặt toàn bộ ngang trẻ 9 tuổi người Caucasian, Bắc Mỹ và người Hàn Quốc từ 9-19 tuổi. Chiều cao mặt toàn bộ có mức gia tăng đều đặn ở khớp cắn loại II và III Angle nhưng không có sự sự gia tăng ở loại I Angle, như vậy loại II và III Angle hàm dưới có xu hướng tăng trưởng xuống dưới nhiều hơn so với loại I Angle.

Bảng 4.1: So sánh chiều cao mặt toàn bộ một số tác giả ( $\left.{ }^{\circ}\right)$

\begin{tabular}{|c|c|c|c|c|c|}
\hline Giới tính & Ricketts [1] & p & Trương Đình Khởi & p & Eun Ju Bae[8] \\
\cline { 1 - 6 } Nam & $60,00 \pm 3,00$ & $>0,05$ & $60,68 \pm 5,25(n=104)$ & $>0,05$ & $62,30 \pm 3,40(n=18)$ \\
\cline { 1 - 1 } & $(n=20)$ & $>0,05$ & $60,41 \pm 4,94(n=102)$ & $>0,05$ & $63,30 \pm 2,60(n=13)$ \\
\hline
\end{tabular}

Kết quả nghiên cứu phù hợp với nghiên cứu của Lê Nguyên Lâm (2014) [6] và của Lê Võ Yến Nhi, Hoàng Tử Hùng (2011) [5] tương ứng với khớp cắn loại I Angle thì không có sự thay đổi theo tuổi có ý nghĩa thống kê. Mức gia tăng tứ 7-9 tuổi (2 năm) loại II Angle (nam: $1,07 \pm 0,42^{\circ}$; nữ: $1,09 \pm 0,43^{\circ}$ ), loại III Angle (nam: 1,92 $\pm 0,29^{\circ}$; nữ: $1,95 \pm 0,27^{\circ}$ ), mức tăng hai giới ngang nhau tương ứng với mối loại khớp cắn, từ 8-9 tuổi có xu hướng tăng nhiều hơn từ 7-8 tuổi nhưng sự khác biệt không có ý nghĩa thống kê (biểu đồ 4.1).
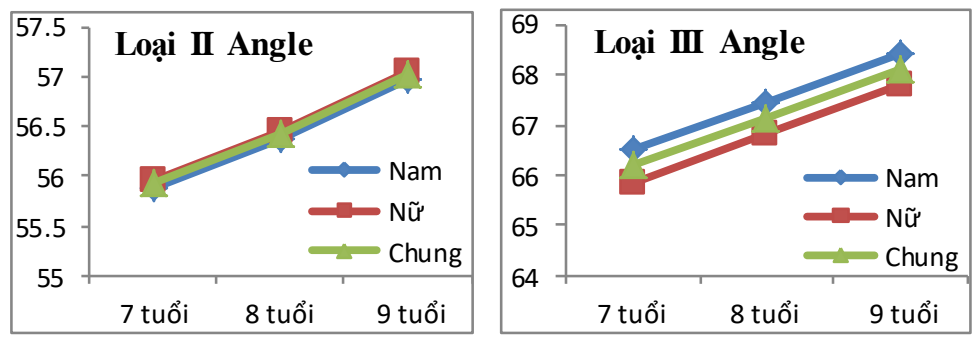

Biểu đồ 4.1: Biểu đồ tăng trưởng chiều cao măt toàn bộ từ 7-9 tuổi

Chiêu cao mặt dưới. Theo kết quả bảng 3.1, chiều cao mặt dưới sự khác biệt không có ý nghĩa thống kê giữa hai giới nhưng ở loại III Angle lớn hơn loại I Angle và loại I Angle lớn hơn loại II Angle, loại III Angle có xu hướng góc mở hơn so với loại I và II Angle theo phân loại khớp cắn của Angle.

\begin{tabular}{|c|c|c|c|c|}
\hline $\begin{array}{l}\text { Chiều cao } \\
\text { mặt dưới }\left({ }^{\circ}\right)\end{array}$ & \begin{tabular}{|c|} 
Tae Soo Park[3] \\
7 tuối
\end{tabular} & $\begin{array}{c}\text { Lế Võ Yến Nhi[5] } \\
10 \text { tuối }\end{array}$ & $\begin{array}{l}\text { Lế Nguyên Lâm[6] } \\
12 \text { tuối }\end{array}$ & $\begin{array}{c}\text { Trương Đình Khởi } \\
7 \text { tuổi }\end{array}$ \\
\hline Nam & $50,10 \pm 3,30(n=40)$ & $46,61 \pm 4,20(n=19)$ & $50,43 \pm 4,94(n=50)$ & $46,13 \pm 4,54$ \\
\hline $\mathrm{p}$ & $<0,05$ & $>0,05$ & $<0,05$ & $(n=104)$ \\
\hline Nữ & $49,40 \pm 4,30(n=50)$ & $45,98 \pm 4,23(n=20)$ & $51,21 \pm 5,17(n=55)$ & $46,23 \pm 5,05$ \\
\hline $\mathrm{p}$ & $<0,05$ & $>0,05$ & $<0,05$ & $(n=102)$ \\
\hline
\end{tabular}

Theo kết quả nghiên cứu của Lê Võ Yến Nhi, Hoàng Tử Hùng (2011) [5] thực hiện trên lứa tuổi từ 10-14 tuổi, giá trị trung bình chiều cao mặt dưới tương tự kết quả trong nghiên cứu của nghiên cứu của Lê Nguyên Lâm (2014) [6] thì có giá trị trung bình lớn hơn, sự khác biệt này có thể do cách chọn mẫu có khác nhau. Tuy vậy, 
các tác giả đều nhận xét rằng chiều cao mặt dưới không gia tăng theo tuổi, kết quả này tương đồng với kết quả loại I Angle, nhưng loại II và III Angle vẫn có sự gia tăng theo tuổi, theo đó loại III có mức gia tăng và tỳ lệ gia tăng lớn hơn loại II Angle. So sánh với kết quả nghiên

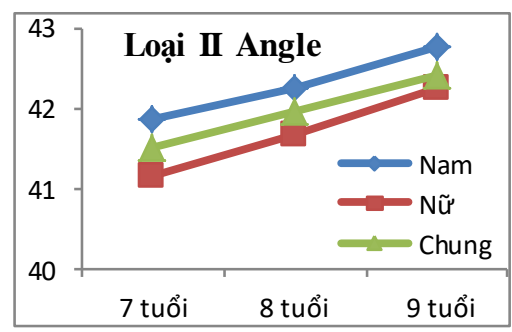

Biểu đồ 4.2: Biểu đồ tăng trưởng

Mức gia tăng từ 7-9 tuổi (2 năm) loại II Angle (nam: $0,90 \pm 0,36^{\circ} ;$ nữ: $1,12 \pm 0,29^{\circ}$ ), loại III Angle (nam: $1,78 \pm 0,40^{\circ} ;$ nữ: $1,79 \pm 0,33^{\circ}$ ), nữ có xu hướng tăng lớn hơn nam, từ 8-9 tuổi có xu hướng tăng lớn hơn từ 7-8 tuổi nhưng sự khác biệt không có ý nghĩa thống kê. Nghiên cứu của Phạm Cao Phong (2016) [7] thực hiện độ tuổi từ 11-13 tuổi, kết quả cho thây nhịp độ tăng trưởng giống nhau giữa nam và nữ nhưng lứa tuổi từ 12 đến 13 hầu hết các kích thước và chỉ số đầu mặt của nam tăng nhanh hơn nữ, tốc độ tăng trưởng đầu mặt từ 12 đến 13 , nam tăng nhanh hơn nữ. Kết quả này khác với lứa tuổi 7-9 tuổi trong nghiên cứu của chúng tôi, khi nhận thấy rằng chỉ số đo đầu mặt ở nữ lớn hơn ở nam và xu hướng tăng trưởng ở nữ sớm hơn nam.

Góc mặt phẳng hàm dưới. Theo kết quả bảng 3.1, góc mặt phẳng hàm dưới bộ sự khác biệt không có ý nghĩa thống kê giữa hai giới cứu của Ricketts [1] và nghiên cứu của Hideyuki Kato, Satoshi Fujii [4] chiều cao mặt dưới ở trẻ 7 tuổi người Kinh tương đương với trẻ 9 tuổi Caucasian, Bắc Mỹ và người Nhật Bản 7 tuổi nhưng nhỏ hơn so với người Hàn Quốc 7 tuổi trong nghiên cứu của Tae Soo Park [3].

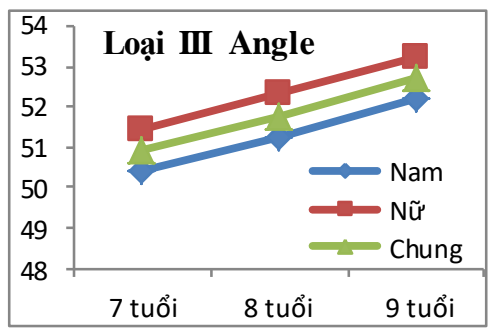

g chiều cao mặt dưới từ 7-9 tuổi

nhưng ở loại III Angle lớn hơn loại I Angle và loại I Angle lớn hơn loại II Angle, loại III Angle có xu hướng góc mở hơn so với loại I và II Angle, sự thay đổi không tương đồng giữa ba nhóm khớp cắn từ 7-9 tuổi, khớp cắn loại I Angle thì góc mặt phẳng hàm dưới giảm theo tuổi trong khi loại II và loại III Angle thì tăng theo tuổi. So sánh giá trị góc mặt phẳng hàm dưới nghiên cứu của Eun Ju Bae, Hye Jin Kwon, Oh Won Kwon (2014) [8], nghiên cứu của Lê Võ Yến Nhi, Hoàng Tử Hùng (2011) [5] và nghiên cứu của Lê Nguyên Lâm (2014) [6] kết quả thấy rằng góc mặt phẳng hàm dưới ở trẻ 7 tuổi người Kinh tương đồng với giá trị đo được ở trẻ 10 tuổi và người Hàn Quốc từ 9-19 tuổi nhưng nhỏ hơn so với trẻ 12 tuổi trong nghiên cứu của Lê Nguyên Lâm, sự khác biệt có thể do cách chọn mẫu khác nhau khi cách chọn mẫu của chúng tôi có tỷ lệ ba nhóm khớp cắn là tương đương nhau.
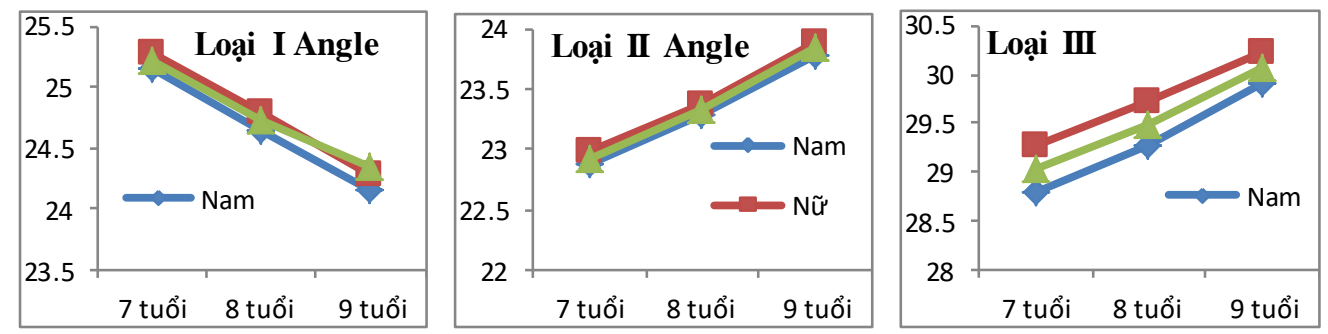

Biểu đồ 4.3: Biểu đồ tăng trưởng góc măt phẳng hàm dưới từ 7-9 tuổi Bảng 4.3: So sánh góc mặt phẳng hàm dưới một số tác giả

\begin{tabular}{|c|c|c|c|c|}
\hline $\begin{array}{c}\text { Góc mă̆t } \\
\text { phằng hàm }\end{array}$ & $\begin{array}{c}\text { Eun Ju Bae } \\
{[8]}\end{array}$ & $\begin{array}{c}\text { Lê Võ Yến Nhí } \\
\text { [5] }\end{array}$ & $\begin{array}{c}\text { Lê Nguyên Lâm } \\
\text { [6] }\end{array}$ & $\begin{array}{c}\text { Trương Đình } \\
\text { Khởi }\end{array}$ \\
\hline dưới ( $\left.{ }^{\circ}\right)$ & 9-19 tuối & 10 tuối & 12 tuối & 7 tuối \\
\hline Nam & $27,00 \pm 5,50(n=18)$ & $27,74 \pm 5,11(n=19)$ & $21,86 \pm 7,19(n=50)$ & $25,54 \pm 3,85$ \\
\hline$p$ & $>0,05$ & $>0,05$ & $<0,05$ & $(n=104)$ \\
\hline Nữ & $27,50 \pm 6,10(n=13)$ & $28,00 \pm 5,97(n=20)$ & $21,53 \pm 6,34(n=55)$ & $25,79 \pm 3,96$ \\
\hline $\mathrm{p}$ & $>0,05$ & $>0,05$ & $<0,05$ & $(n=102)$ \\
\hline
\end{tabular}


Theo kết quả nghiên cứu, góc mặt phẳng hàm dưới ở loại I Angle giảm dần theo tuổi, mức giảm tương ứng của nam là $-0,99 \pm 0,38^{\circ}$, của nữ là $-1,01 \pm 0,39^{\circ}$; phù hợp với nghiên cứu của Ricketts R.M (1996) [1] khi đưa ra nhận xét rằng góc mặt phẳng hàm dưới giảm trung bình 0,5 $\left({ }^{\circ}\right) /$ năm. Tuy nhiên, loại II và III Angle có mức gia tăng theo tuổi, nữ có xu hướng tăng lớn hơn nam, từ 8-9 tuổi có xu hướng tăng lớn hơn từ 78 tuổi nhưng sự khác biệt không có ý nghĩa thống kê.

\section{KẾT LUÂN}

Qua nghiên cứu dọc thực hiện bằng phương pháp đo trên ảnh chuẩn hóa thẳng và nghiêng nhân trắc đầu - mặt trên 206 trẻ em 7 -9 tuổi người Kinh (104 nam, 102 nữ), rút ra kết luận sau: Chiều cao mă̆t toàn bộ, chiều cao tầng mặt dưới, góc mă̆t phẳng hàm dưới giống nhau giữa nam và nữ ở trẻ 7 tuổi, ở loại III lớn hơn loại I và II Angle. Chiêu cao mặt toàn bộ và chiều cao mặt dưới không đổi ở loại I Angle, tăng trưởng đều đăn ở loại II và III Angle từ 7-9 tuổi. Góc mặt phẳng hàm dưới giảm theo tuổi ở loại I Angle, tăng dần theo tuổi ở loại II và III Angle. Nữ có xu hướng tăng trưởng sớm hơn nam.

Lời cảm ơn. Trân trọng cảm ơn những đối tượng tự nguyện tham gia nghiên cứu, cảm ơn sự giúp đõ̃ của Ban giám hiệu trường Tiểu học Liên Ninh, Thanh Trì, Hà Nội. Xin chân thành cảm ơn Viện Đào tạo Răng Hàm Mặt - Đại học Y Hà Nội đã tạo điều kiện cho chúng tôi hoàn thành nghiên cứu này.

\section{TÀI LIÊU THAM KHẢO}

1. Ricketts RM (1996). Progressive cephalometrics paradigm 2000, American Institute for Bioprogressive Education, Scottsdale, Arizona.

2. Platou C, Zachrisson B. U (1983). Incisor position in Scandinavian children with ideal occlusion, Am J Orthod, 83(4), 341-352.

3. Tae Soo Park (1984). A longitudinal cephalometric study of craniofacial growth of Korean children, Korean Journal of Orthodontics, 14(2), 217-231.

4. Hideyuki Kato, Satoshi Fujii (1988). Application of the Ricketts Analysis to Children in the Primary Dentition: Second Report: A Study of Annual Growth, The Japanese Journal of Pediatric Dentistry, 26, 755-768.

5. Lê Võ Yến Nhi, Hoàng Tử Hùng (2011). Sứ tăng trưởng so măt ở trẻ em Việt Nam từ $10-14$ tuổi theo phân tích Ricketts. Y học Thành phố Hồ Chí Minh, 15(2), 21-30.

6. Lê Nguyên Lâm, Nguyễn Bắc Hùng (2014). Sự tăng trưởng của xương hàm dưới ở trẻ từ 12 15 tuổi theo phân tích Ricketts, Tạp chí Y học thực hành, 6(923), 67-71.

7. Pham Cao Phong, Lê Gia Vinh (2016), Sư phát triển và sự xoay của xương hàm dưới lứa tuổi 1112 trên phim sọ nghiêng, Tạp chí Y Học Việt Nam, 453(1), 7-9.

8. Eun-ju Bae (2013). Changes in longitudinal craniofacial growth in subjects with normal occlusions using the Ricketts analysis, The Korean Journal of Orthodontics, 71-79.

\title{
ĐIỀU TRI TIÊU HUYẾT KHỐI TĨNH MACH Ở BÊNH NHÂN NHỒI MÁU NÃO CẤP KHÔNG XÁC ĐİNH CHÍNH XÁC THỜI ĐIỂM KHỞI PHÁT DỰA VÀO MISMATCH DWI - FLAIR TRÊN MRI SỌ NÃO
}

\author{
Nguyễn Thị Hoa ${ }^{1}$, Nguyễn Tiến Dũng ${ }^{2}$, \\ Trần Anh Tuấn ${ }^{2}$, Đào Việt Phương ${ }^{2}$, Mai Duy Tôn ${ }^{2}$
}

\section{TÓM TẮT}

Mục tiêu: Mô tả đặc điểm lâm sàng, hình ảnh học và kết quả điều trị tái tưới máu bằng thuốc tiêu huyết khối đường tĩnh mạch ở bệnh nhân đột quỵ nhồi máu não cấp không xác định chính xác thời điểm khởi phát dựa vào hình ảnh không phù hợp DWI - FLAIR trên phim chụp MRI sọ não. Phương pháp nghiên cứu: Hồi cứu mô tả loạt ca bệnh, theo dõi kết cục lâm sàng

\footnotetext{
${ }^{1}$ Bệnh viện Thanh Nhàn

${ }^{2}$ Bềnh viện Bach Mai

Chịu trách nhiệm chính: Nguyễn Thị Hoa

Email: hoaphuong972008@gmail.com

Ngày nhận bài: 10.6.2021

Ngày phản biên khoa hoc: 3.8.2021

Ngày duyệt bài: 13.8.2021
}

tới 90 ngày sau khởi phát. Đối tượng là các bênh nhân từ 18 tuổi trở lên, đôt quy nhồi máu não cẩp không xác định chính xác thời điểm khởi phát được điều trị bằng thuốc tiêu huyết khối alteplase đường tĩnh mach tại Bệnh viện Bach Mai, đáp ứng yêu cầu khoảng thời gian từ thời điểm cuối cùng bình thường tới khi được tiêu huyết khối trên 4,5 giờ, và khoảng thời gian từ khi được phát hiện đột quỵ tới tiêu huyết khối là dưới 4,5 giờ, có hình ảnh không tương xứng DWI - FLAIR trên phim chup MRI so não, loai trừ những bệnh nhân được chỉ định lấy huyết khối cơ học. Kết quả: Từ tháng 5/2019 tới tháng 5/2021 có 40 bệnh nhân đáp ứng đủ điều kiện nghiên cứu. $72.5 \%$ là nam, tuổi trung bình 67.05 tuối, $75 \%$ được phát hiện đột quy. khi thức giấc. Thời gian trung bình từ lần cuối còn bình thường tới khi được dùng thuốc tiêu huyết khối là 7.75 giờ. Thời gian trung bình từ khi phát hiện đột quy. 